EPJ manuscript No.

(will be inserted by the editor)

\title{
An introduction to the Generalized Parton Distributions
}

\author{
Michel Garçon \\ DAPNIA/SPhN, CEA-Saclay, 91191 Gif-sur-Yvette, France \\ Received: date / Revised version: date
}

\begin{abstract}
The concepts of Generalized Parton Distributions (GPD) are reviewed in an introductory and phenomenological fashion. These distributions provide a rich and unifying picture of the nucleon structure. Their physical meaning is discussed. The GPD are in principle measurable through exclusive deeply virtual production of photons (DVCS) or of mesons (DVMP). Experiments are starting to test the validity of these concepts. First results are discussed and new experimental projects presented, with an emphasis on this program at Jefferson Lab.
\end{abstract}

PACS. 12.38.-t Quantum chromodynamics - 13.60.-r Photon and charged-lepton interactions with hadrons

In Memoriam : René Beurtey died a few days before this conference began. His work has had a determining influence on the technical and physical developments in our field. His ideas were innovative and he shared them generously, not being concerned with "publishing first". He developed the first adiabatic transitions in a polarized deuteron source, promoted original technics for the study of nucleon-nucleon scattering as a continuous function of energy (cluster jet inside a synchrotron ring and the "Beurtey-Saudinos" wheel), and triggered the "Tatischeff experiments" on narrow dibaryons search. In addition, he participated in the accelerator research that made SATURNE the first synchrotron to successfully cross proton depolarizing resonances. Beyond his professional talents, René had an expansive and enjoyable personality that made him a true pleasure to work with or around, or to consult. His lively wit and sense of humor were a stimulation for many of us.

\section{Foreword}

This presentation is intended to provide a pedagogical introduction to the subject of Generalized Parton Distributions and associated phenomenology. It cannot be exhaustive on the subject, and the reader should consult reviews or papers such as Refs. [1, 2, 3, [1, [3], and references therein, for a more rigourous and complete account of the subject. At this conference, M. Vanderhaeghen gave another talk on the subject, with more emphasis on theory and its latest developments [6].

\section{Basic concepts}

In the last few years, significant theoretical advances were made that allow, using Quantum Chromodynamics (QCD)

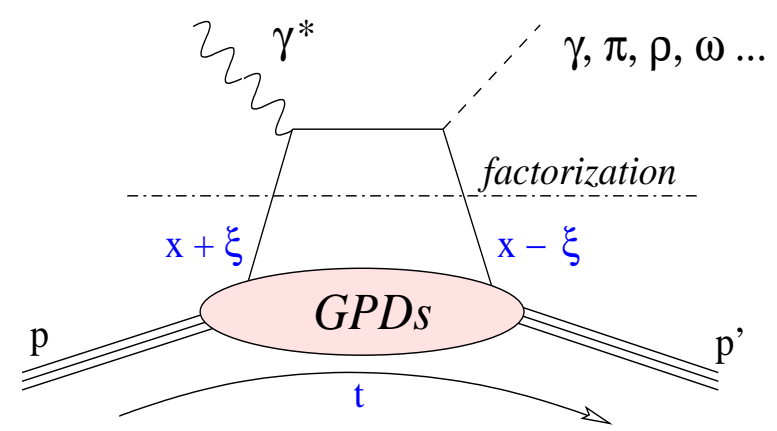

Fig. 1. Representation of the deeply virtual exclusive processes DVCS and DVMP, also referred to as the "handbag" diagram.

and within some conditions, to factorize the amplitude for exclusive electroproduction processes into a hard scattering at the parton level and non-perturbative Generalized Parton Distributions (GPD) - see Fig. 11. From the theoretical point of view, as a particular case of factorization theorems, the introduction of these distributions builds a bridge between fundamental QCD, phenomenology and experimental observables. The processes under consideration are the deeply virtual Compton scattering (DVCS: $e p \rightarrow e p \gamma$ ) and the deeply virtual meson production (DVMP: $e p \rightarrow e p \rho, \omega, \pi \ldots$ ). We understand by "deeply virtual" a kinematical regime where the virtuality $Q^{2}$ of the exchanged photon $\gamma^{*}$ is large and where the available energy in the $\gamma^{*} p$ system is above the resonance region, typically larger than $2 \mathrm{GeV}$.

The GPDs contain the hitherto most complete description of the internal quark-gluon structure of hadrons. For each of the quark flavours (we will not consider the gluon sector in this talk), there are four of these distributions, $H, E, \tilde{H}$ and $\tilde{E}$, which depend on three variables: the average longitudinal momentum fraction $x$ of the struck par- 
ton in the initial and final states, a skewness parameter $\xi$ which measures the difference between these two momentum fractions, and the momentum transfer $t$ to the target nucleon. The above mentioned factorization theorems [7, 8] are applicable for $|t| / Q^{2} \ll 1$.

The physical content of the GPDs is quite rich: in simple terms, whereas ordinary parton distributions yield, for example, the probability $|\psi(x)|^{2}$ that a quark carries a fraction $x$ of the momentum of a fast moving nucleon, the GPDs measure the coherence $\psi^{*}(x-\xi) \cdot \psi(x+\xi)$ between two different (quark) momentum states of the nucleon and in this way quark momentum correlations in the nucleon. In quantum mechanics, one cannot reduce the knowledge of a system formed of a superposition of states (in our case, an infinite number of states described by the variable $x$ ) to the sole probabilities that the system be in a particular state. The interferences between these states, or coherences, or off-diagonal elements, are necessary for a full description of the system.

If one of the momentum fractions $(x+\xi$ or $x-\xi)$ is negative, it is interpreted as representative of an antiquark. Meson-like $q \bar{q}$ configurations in the nucleon may then be investigated. As mentioned in Sec. 3.2, the GPDs are expected to exhibit a rich structure at $|x|<\xi$ which is related in particular to the pion cloud around the nucleon.

Whereas $x$ and $\xi$ characterize solely the longitudinal momenta of the partons involved, the $t$-dependence of the GPDs is related to their transverse momenta. By Fourier transform, it is conceivable to access simultaneously the longitudinal momentum fraction of quarks and their position in the transverse plane [9]. This would open the way to a femto-photography of the nucleon 10 .

The richness of information contained in the GPDs may be illustrated by several quite remarkable relations, among which

- the forward limit:

$$
\begin{gathered}
\lim _{t \rightarrow 0, \xi \rightarrow 0} H(x, \xi, t)=q(x), \text { or }-\bar{q}(-x) \text { if } x<0 \\
\text { and } \\
\lim _{t \rightarrow 0, \xi \rightarrow 0} \tilde{H}(x, \xi, t)=\Delta q(x), \text { or } \Delta \bar{q}(-x) .
\end{gathered}
$$

As expected from the above discussion, the ordinary parton distributions, both unpolarized $q(x)$ and polarized $\Delta q(x)$, are but a limiting case of the GPDs. Note that, though the GPDs are defined functions for $\xi=0$ or $t=0$, these variables take only finite, non-zero values in any experiment. Note also that the functions $E$ and $\tilde{E}$ have no connection with the ordinary parton distributions. They are not constrained by deeply inelastic scattering, which corresponds to this forward limit.

- the form factor limit:

$$
\forall \xi, \quad \int_{-1}^{+1} H(x, \xi, t) \cdot d x=F(t)
$$

(4 relations of this type for each quark flavor). After integration over $x$, the $t$-dependence of the GPDs is given by the nucleon elastic form factors $(H \rightarrow$ Dirac, $E \rightarrow$ Pauli, $\tilde{H} \rightarrow$ axial vector and $\tilde{E} \rightarrow$ pseudoscalar form factors).

- Ji's sum rule:

$$
\forall \xi, \quad \lim _{t \rightarrow 0} \frac{1}{2} \int_{-1}^{+1} x \cdot(H+E)(x, \xi, t) \cdot d x=J_{q},
$$

where $J_{q}$ is the total angular momentum, i.e. the sum of intrinsic spin and of orbital angular momentum, carried by the quarks. This sum rule provides a way to understand the origin of the nucleon spin. Indeed, the contribution of the quark intrinsic spin $\left(\frac{1}{2} \Delta \Sigma\right)$ was measured at CERN/SMC, SLAC and DESY/HERMES. The gluon contribution $(\Delta G)$ will be determined at CERN/COMPASS, RHIC/STAR and SLAC. The knowledge of GPDs allows one to isolate the contribution of the quark orbital angular momentum to the total spin of the nucleon: $\frac{1}{2}=J_{q}+J_{g}=\left(\frac{1}{2} \Delta \Sigma+L_{q}\right)+$ $\left(\Delta G+L_{g}\right)$.

The first two relations demonstrate the new unifying frame given by the GPDs: observables as different as elastic form factors and parton distributions are related to each other through these new functions. However, the knowledge of limits, or projections, does not imply a complete knowledge of the GPDs themselves. Models are being developed for a complete representation.

\section{GPD calculations}

We give here a short summary of selected calculations of the GPDs. For a more complete account, see Refs. [3, 5, 6].

\subsection{Constrained parametrizations}

The "double distributions" [1] are well suited for the description of the GPDs. They incorporate the GPDs mathematical properties (polynomiality of the Mellin moments) and have a definite physical content. They satisfy positivity bounds, a number of inequalities that GPDs have been shown to obey. Elastic form factors and parton distributions are included as limiting values.

An alternate, "dual" parametrization, based on a partial wave expansion, has recently been proposed [12].

\subsection{Model calculations}

QCD-inspired models are very useful to understand how non-perturbative mechanisms generate various structures in GPDs.

In the simple bag model, GPDs have but a weak dependence on $\xi$, and show no structure for $|x|<\xi$. A number of calculations in different constituent quark models are being developed.

In contradistinction, in the chiral quark-soliton model, where the quarks interact through a highly non-linear pion 
Table 1. Comparative merits of DVCS and DVMP for the study of Generalized Parton Distributions

\begin{tabular}{|c|c|}
\hline DVCS & DVMP \\
\hline $\begin{array}{c}\text { Factorization established } \\
\text { (virtual photon is transverse at leading order) }\end{array}$ & $\begin{array}{c}\text { Factorization established for longitudinal photons } \\
\left(\sigma_{L} \text { must be extracted experimentally }\right)\end{array}$ \\
\hline Measures a combination of $H, E, \tilde{H}, \tilde{E}$ & $\begin{array}{l}\text { Vector mesons : combination of } H \text { and } E \\
\text { (the GPD's entering Ji's sum rule) } \\
\text { Pseudoscalar mesons : combination of } \tilde{H} \text { et } \tilde{E} \\
\text { Various decompositions in quark flavours } \\
\text { according to the nature of the meson }\end{array}$ \\
\hline$\sigma_{T}\left(\gamma^{*} p \rightarrow \gamma p\right) \propto Q^{-4}$ & $\begin{array}{c}\sigma_{L}\left(\gamma^{*} p \rightarrow p \text { meson }\right) \propto Q^{-6} \\
\left.\text { (larger at least for } \pi^{+}, \rho \text { and } \omega \text { at moderate } Q^{2}\right)\end{array}$ \\
\hline \multicolumn{2}{|l|}{$\begin{array}{c}\text { Contribution of Bethe-Heitler process: } \\
\text { - useful through interferences } \\
\text { (polarization or charge asymmetries measurements) } \\
\text { - decreases quickly with increasing energy }\end{array}$} \\
\hline & A manageable complication: the meson structure \\
\hline Smaller higher twist contributions & $\begin{array}{l}\text { Larger higher twist contributions, but cancel } \\
\text { in ratios } \rho / \omega \text { and polarization asymmetries }\end{array}$ \\
\hline
\end{tabular}

field, the GPDs exhibit a rich structure for $|x|<\xi$. The same model leads also to a $t$-dependence which can be approximated by $H(x, 0, t) \sim x^{-a t} q(x)$ and deviates significantly from the early factorized ansatz $H(x, 0, t) \sim$ $F(t) q(x)$.

In the region $x>\xi$, the use of light-cone wave functions for the nucleon allows to write GPDs as overlap integrals. In considering the nucleon as a superposition of Fock states, e.g. $|p>=| u u d>+|u u d q \bar{q}>+| u u d g>+\cdots$, the GPDs are useful to understand the role of given states.

\subsection{Lattice QCD}

Moments of ordinary parton distributions are being evaluated using QCD calculations on a lattice. Likewise, GPD moments can be calculated [13].

\section{Observables}

Both DVCS and DVMP processes are sensitive to GPDs of the nucleon, once it is established that the handbag diagram of Fig. 1 dominates in the kinematical regions where measurements are performed. These processes have different merits and sensitivities summarized in Table 11. The sensitivity of specific observables, including polarization observables, has been studied in detail in Refs. [3, 5].

While $\xi$ and $t$ are variables fixed by the kinematics of the process under study, $x$ enters the amplitude as an integration variable. Therefore, in most cases, observables are expressed in terms of integrals over $x$ of GPDs. The only exception is in observables sensitive only to the imaginary part of the handbag amplitude: because of the propagators $(x \pm \xi+i \varepsilon)^{-1}$ in the diagram of Fig. 1, the GPDs enter then with an argument $x= \pm \xi$. This is the case for the DVCS beam spin asymmetries discussed in Sec. 6 .

In general, the extraction of GPDs from observables requires a deconvolution and, in principle, an infinite set of data. In practice, this problem has mathematical properties analogous to e.g. tomography 14 and may be solved (with associated uncertainties) with a finite set of data. This complete exercise remains to be done with either real or simulated data.

\section{Finite $Q^{2}$}

Most of the discussion so far was based on a high momentum transfer approximation (Bjorken limit). In practice, the measurements of the exclusive processes under study are or will be performed at rather moderate $Q^{2}, 1$ to 6 $\mathrm{GeV}^{2}$. The relevant corrections are listed in this Section.

As is the case for the ordinary parton distributions, the GPD evolution leads to logarithmic corrections $\left(\ln Q^{2}\right)$ : the dependence of the GPDs on a factorization scale $\mu$ results from the evolution equation

$\mu \frac{\partial}{\partial \mu} H(x, \xi, t ; \mu)=\int \mathcal{K}\left(x, y, \xi ; \alpha_{s}(\mu)\right) \cdot H(y, \xi, t ; \mu) \cdot d y$, where $\alpha_{s}$ is the strong coupling constant. The kernel $\mathcal{K}$ has been calculated at next-to-leading order (NLO).

At order $\mathcal{O}(1 / Q)$ (twist-3), beyond a minimal gauge restoring term, the contribution of a longitudinal exchanged photon $\gamma_{L}^{*}$ to DVCS can be expressed in terms of derivatives of (twist-2) GPDs. This is the so-called Wandzura-Wilczek approximation. The exchange of a gluon between the quark in the handbag loop and the nucleon core would give an additional contribution, thought to be very small, in analogy with a similar case in deeply inelastic scattering.

At the next order in $Q$, trivial kinematical corrections $\mathcal{O}\left(t / Q^{2}, M^{2} / Q^{2}\right)$, surprisingly enough, are not always considered. There are also dynamical target mass corrections $\mathcal{O}\left(M^{2} / Q^{2}\right)$. Finally the transverse momenta of quarks modify the quark propagators in the diagram of Fig. 1: $(x \pm \xi+i \varepsilon)^{-1} \rightarrow\left(x \pm \xi+k_{\perp}^{2} / Q^{2}+i \varepsilon\right)^{-1}$. Many other twist- 4 contributions remain to be evaluated.

The aim of the first generation experiments (see Sec. 6) is then to establish that observables have the anticipated 
Michel Garçon: An introduction to the Generalized Parton Distributions

$Q^{2}$ dependence in order to validate the GPD approach at leading twist or to disentangle higher twist contributions.

\section{Experiments}

The difficulty in the experimental study of GPDs is the measurement of exclusive processes, of rather low cross sections, at the highest possible momentum transfer $Q^{2}$. This requires high beam energy and luminosity, as well as large acceptance and high resolution detectors. At present, these studies have been initiated at HERMES (sufficient energy, $27 \mathrm{GeV}$, but relatively low luminosity) and at Jefferson Lab (beam energy of 4 to $6 \mathrm{GeV}$, but high product luminosity $\times$ acceptance). In the next few years, significant progress could be achieved with a $12 \mathrm{GeV}$ upgrade at JLab/CEBAF 15, and using the $200 \mathrm{GeV}$ muon beam at COMPASS 16. A new high luminosity, high duty cycle machine, operating in the $25-50 \mathrm{GeV}$ range, would be ideally suited for these studies 17].

DVCS should be the cleanest process for GPD studies. Through the optical theorem, it is linked to deeply inelastic scattering for which the evolution of the structure functions is well understood down to $Q^{2} \sim 1 \mathrm{GeV}^{2}$. In addition, the $\gamma \gamma^{*} \pi^{0}$ form factor, whose calculation proceeds through the same type of handbag diagram as in Fig. 1, gives also a good indication that DVCS should scale early. Corrections may then be expected to be understood at rather low $Q^{2}$. As mentioned in Table 1, DVCS is indistinguishable from the known Bethe-Heitler $(\mathrm{BH})$ process where the final photon is emitted by the incident or scattered electron. DVCS dominates over BH only at very high energies (COMPASS, HERA). At beam energies accessible at JLab or HERMES, the large BH process may be turned into an advantage by measuring interferences in spin or charge asymmetries; DVCS could then be accessed at the amplitude level.

The first experimental indications of DVCS at the quark level were published last year [18, 19]. DVCS was also observed at HERA [20], in a kinematical region that makes it more sensitive to gluons. The approximate $\sin \Phi$ dependence of the observed beam spin asymmetry (see Fig. 2) is expected from any process which conserves helicity in the $\gamma^{*} p \rightarrow \gamma p$ scattering 22]. The process described by the (leading order) handbag diagram of Fig. 1 1 has such a property. The comparison with first calculations [3,5, 21] using the GPD formalism is very encouraging. From the experimental point of view, it should be noted that at HERMES, the $800 \mathrm{MeV}$ missing mass resolution in the $e p \rightarrow e \gamma X$ reaction is not sufficient to select unambiguously the (dominating) $e p \rightarrow e p \gamma$ events, while at JLab/CLAS, the 110 $\mathrm{MeV}$ missing mass resolution in the $e p \rightarrow e p X$ reaction causes the $e p \rightarrow e p \gamma$ and $e p \rightarrow e p \pi^{0}$ to mix, but a separation was done using a shape analysis of the relevant spectra. First results of beam charge asymmetry measurement in DVCS were also reported from HERMES [23].

Both experiments will soon obtain more statistics. Furthermore, both detection systems will be improved in the next couple years. In particular, at JLab/CLAS, a forward lead-tungstanate calorimeter, covering the photon

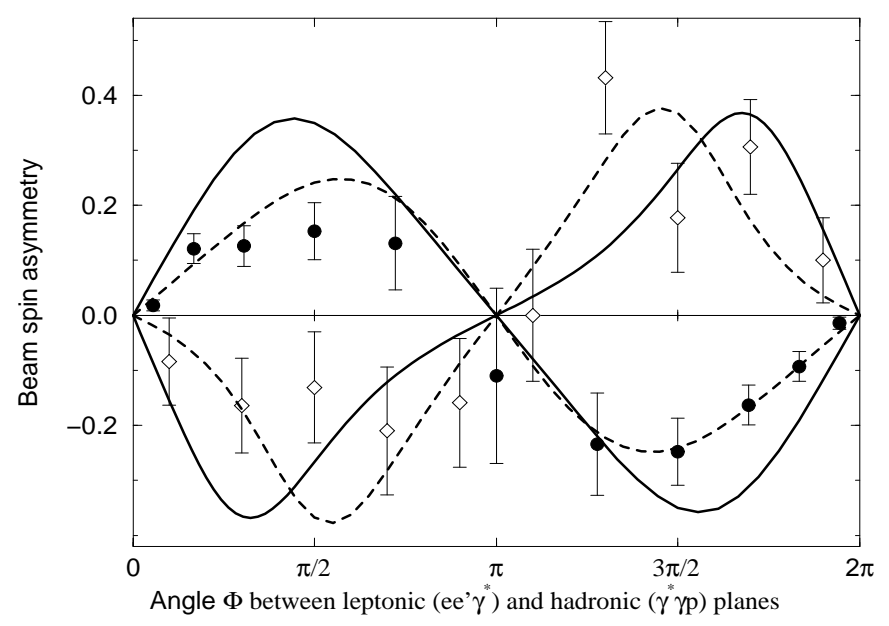

Fig. 2. Beam spin asymmetry in $\mathbf{e} p \rightarrow e p \gamma$. The diamonds are results from HERMES [18], the full circles from JLab/CLAS [19]. The kinematics are different for the two experiments. The corresponding calculations are from Refs. [3] (solid lines) and [5] (dashed lines). The asymmetry is of opposite sign between the two experiments because one uses a positron beam while the other uses electrons. The approximate $\sin \Phi$ dependence is strongly suggestive of the validity of the GPD approach at leading order.

angles between 3 and $12^{\circ}$, is being built, together with a specially designed two-coil solenoid acting as a trap for background Möller electrons [24]. A luminosity of $2 \times 10^{34}$ $\mathrm{cm}^{-2} \mathrm{~s}^{-1}$ is anticipated with this large acceptance spectrometer. Another DVCS experiment will run in 2003 at JLab/Hall A [25], using one High-Resolution Spectrometer for detection of the scattered electrons, a new leadfluoride calorimeter for the outgoing photons, and a ring of thick plastic scintillators for the recoil protons [25]. Preliminary tests indicate that a luminosity of $10^{37} \mathrm{~cm}^{-2} \mathrm{~s}^{-1}$ can be reached, in spite of the rather open geometry and forward disposition of the proton detector.

Because of the necessary additional gluon exchange to create a meson, the asymptotic regime should be reached at higher $Q^{2}$ in DVMP processes. However observables such as asymmetries and ratios (e.g. $\rho / \omega$ ) are expected to scale earlier. As illustrated in Table i, DVMP, at leading order, is sensitive to different combinations of GPDs as DVCS. Also the different quark content of various mesons gives access to different flavour combinations of these distributions. Since factorization has been proven only for longitudinal photons [8], the corresponding contribution must be extracted from the experiment. For vector mesons, this is done via a measurement of the meson polarization through the angular distribution of its decay products $\left(\rho \rightarrow \pi^{+} \pi^{-}, \omega \rightarrow \pi^{+} \pi^{-} \pi^{0}, \phi \rightarrow K^{+} K^{-}\right)$and the verifiable application of $s$-channel helicity conservation to relate the virtual photon helicity to the one of the emitted vector meson. For pseudoscalar mesons, one has to resort to a Rosenbluth separation to extract $\sigma_{L}$ (this has not been attempted yet in the considered kinematical regime). 


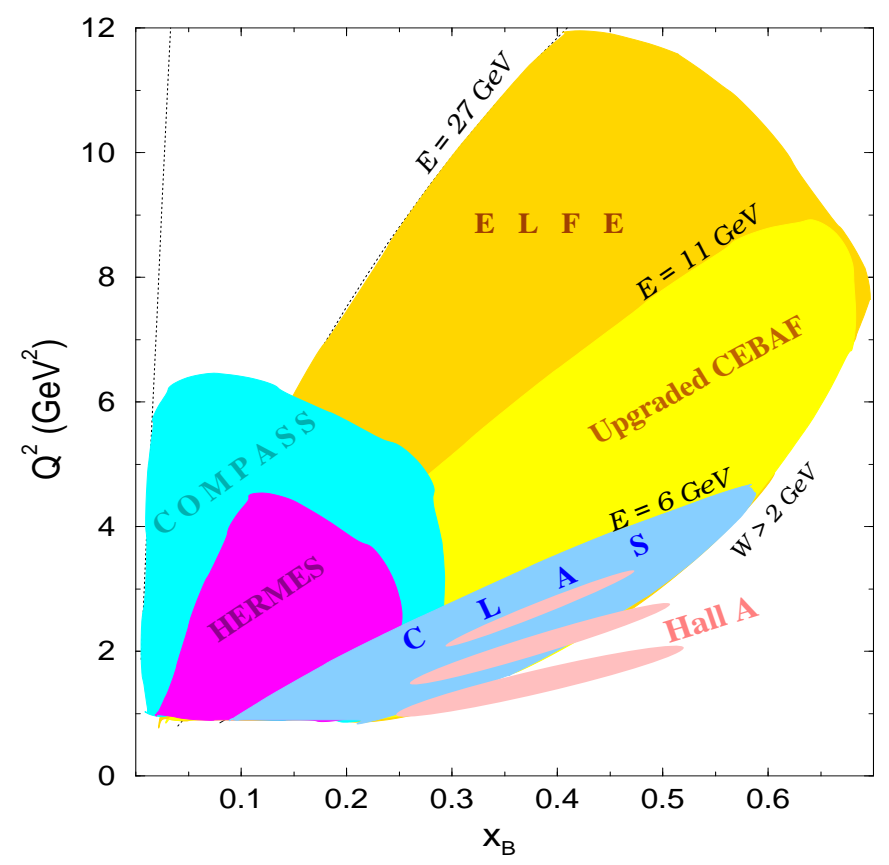

Fig. 3. Kinematical domain $\left(Q^{2}\right.$ vs $\left.x_{B}\right)$ accessible at various facilities (existing or in project) for the measurement of deeply virtual exclusive processes. $x_{B}$ is the usual Bjorken variable, from which $\xi$ is calculated. "ELFE" does not refer here to a specific project, but to a - yet to be defined - high luminosity machine, in this case at $27 \mathrm{GeV}$. See text for additional comments.

The first results of (almost) exclusive electroproduction of $\rho$ mesons (longitudinal cross sections) [26] in a kinematical region where the handbag diagram is expected to dominate are also very suggestive of the validity of the GPD approach. Systematic studies and tests of the predicted $Q^{-6}$ scaling of the longitudinal cross sections are underway at JLab/CLAS: data taken at beam energies of $4.2 \mathrm{GeV}$ [27] and $5.75 \mathrm{GeV}$ [28], up to $Q^{2} \sim 4 \mathrm{GeV}^{2}$, are being analyzed.

The kinematical domain accessible with existing and projected facilities is illustrated in Fig. 3. The high $Q^{2}$ approximate limit for the existing high energy facilities results from count rate limitations due to a lower luminosity and decreasing cross sections as $Q^{2}$ and $x_{B}$ increase.

\section{Conclusions and outlook}

Generalized Parton Distributions have emerged in the past few years as a powerful, attractive and unifying concept for the hadron structure. Their $Q^{2}$ evolution has been worked out to next-to-leading order, and twist-3 (and some twist4) contributions to deeply virtual and exclusive processes have been calculated. The interpretation and significance of GPDs are under intense study.

The deconvolution problem posed by the extraction of GPDs from experimental observables was barely touched upon, but mathematical methods exist, or are being developed, that address this particular issue.
On the experimental side, we are still at a very early stage. There are indications that the handbag diagram is at work. In the next two or three years, more dedicated and higher statistics experiments will attempt to establish on firm grounds the validity of this theoretical approach; this includes tests of $Q^{2}$-scaling laws and of factorization. Beyond that, systematic measurements of GPDs will be performed in a large kinematical domain at several facilities.

\section{References}

1. M. Vanderhaeghen, P.A.M. Guichon and M. Guidal, Phys. Rev. D 60 (1999) 094017; P.A.M. Guichon and M. Vanderhaeghen, Prog. Part. Nucl. Phys. 41 (1998) 125-190.

2. B.W. Filippone and Xiandong Ji, Adv. Nucl. Phys. 26 (2001) 1-88.

3. K. Goeke, M.V. Polyakov and M. Vanderhaeghen, Prog. Part. Nucl. Phys. 47 (2001) 401-515.

4. A.V. Radyushkin, hep-ph/0101225.

5. A.V. Belitsky, D. Müller and A. Kirchner, Nucl. Phys. B629 (2002) 323-392.

6. M. Vanderhaeghen, these proceedings.

7. X. Ji, Phys. Rev. D 55 (1997) 7114-7125; A.V. Radyushkin, Phys. Rev. D 56 (1997) 5524-5557.

8. J.C. Collins, L. Frankfurt, and M. Strikman, Phys. Rev. D 56 (1997) 2982-3006.

9. M. Burkardt, Phys. Rev. D 62 (2000) 071503(R).

10. J.P. Ralston and B. Pire, hep-ph/0110075; M. Diehl, Eur. Phys. Jour. C 25 (2002) 223-232; J.P. Ralston and P. Jain, hep-ph/0207129; M. Burkardt, hep-ph/0209179.

11. A.V. Radyushkin, Phys. Rev. D 59 (1999) 014030.

12. M.V. Polyakov and A.G. Shuvaev, hep-ph/0207153.

13. W. Schroers, Workshop on Hadronic phenomenology from lattice gauge theory, Univ. Regensburg, August 1-3, 2002.

14. O.V. Teryaev, Phys. Lett. B 510 (2001) 125-132.

15. The Science Driving the $12 \mathrm{GeV}$ Upgrade of CEBAF, Jefferson Lab report, February 2001.

16. N. d'Hose et al., Letter of intent to the CSTS/SPhN-Saclay (2000), http://www-dapnia.cea.fr/Sphn/Vcs/Deep/.

17. Declaration of Ferrara, http://www.fe.infn.it/qcd-n02/.

18. A. Airapetian et al. (HERMES collaboration), Phys. Rev. Lett. 87 (2001) 182001.

19. S. Stepanyan et al. (CLAS collaboration), Phys. Rev. Lett. 87 (2001) 182002.

20. C. Adloff et al. (H1 collaboration), Phys. Lett. B517 (2001) 47-58.

21. A. Freund, M. McDermott and M. Strikman, hep$\mathrm{ph} / 0208160$.

22. M. Diehl et al., Phys. Lett. B411 (1997) 193-202.

23. G. van der Steenhoven, these proceedings.

24. V. Burkert, L. Elouadrhiri, M. Garçon, S. Stepanyan et al., CEBAF experiment 01-113.

25. P. Bertin, C. Hyde-Wright, R. Ransome, F. Sabatié et al., CEBAF experiment 00-110.

26. A. Airapetian et al. (HERMES collaboration), Eur. Phys. Jour. C 17 (2000) 389-398.

27. C. Hadjidakis, private communication.

28. M. Guidal, M. Garçon, E. Smith et al., CEBAF experiment 99-105. 